\title{
On the numerical solution of Bagley-Torvik equation via the Laplace transform
}

\author{
Marjan Uddin and Suleman Ahmad \\ Department of Basic Science, University of Engineering and Technology Peshawar, Pakistan. \\ E-mail: marjan@uetpeshawar.edu.pk
}

\begin{abstract}
In this work, we formulate a numerical scheme for the solution of the Bagley-Torvik equation using an integral representation in complex plane. The resultant integral is approximated to high order accuracy using quadrature. The accuracy of numerical algorithm depends on the selection of optimal contour of integration. Several contour have been developed in the literature for solving FDEs. In the present work, we will investigate the applicability of optimal contours for solving Bagley-Torvik equation. We compared our results with other methods available in the literature to validate the efficiency and accuracy of the method for various optimal contour of integrations.
\end{abstract}

2010 Mathematics Subject Classification. 65R10. 65M15

Keywords. Bagley-Torvik equation, Laplace Transform, Quadrature.

\section{Introduction}

The most general form of Bagley-Torvik (BT) equation is,

$$
a_{1} \frac{d^{2} v(t)}{d t^{2}}+b_{1} \frac{d^{\alpha} v(t)}{d t^{\alpha}}+c_{1} v(t)=h(t)
$$

where $a_{1}, b_{1}, c_{1}$ are real numbers, $a_{1} \neq 0, \alpha=\frac{3}{2}$. BT equation was developed in the work of [8], it was further discussed in work of [9]. BT equation uses to model the dynamics of rigid plate in fluid. The most suitable initial conditions used for this model may be specified as,

$$
v^{(k)}(0)=v_{0}^{(k)}, k=0,1, \ldots, m-1, \text { and } m-1<\alpha \leq m .
$$

where the Reimann-Liouville differential operators of order $\alpha>0$, defines as

$$
D^{\alpha} v(t)=\frac{1}{\Gamma(m-\alpha)} \frac{d^{m}}{d t^{m}} \int_{0}^{t} \frac{v(u)}{(t-u)^{\alpha-m+1}} d u,
$$

where the integer $m$ is given as $m-1<\alpha \leq m$. For more detail see for example $[1,2,13,14]$. In the present work, we use laplace transform and quadrature method to approximate the solution of BT equation. We consider how the theoretical results may be applied in practical cases. In particular we consider the performance of existing numerical methods for solving Bagley-Torvik equation when the equation to be solved depend upon parameters that must be estimated and are subject to errors. 
Definition 1.1. If $c(>-1) \in \mathbb{R}$ then:

$$
£\left[t^{c}\right]=\frac{\Gamma(c+1)}{s^{c+1}}, \quad \text { where } R(s)>0 .
$$

Lemma 1.2. The expression for the Laplace transformation of fractional order differential operator, when $m-1<\alpha \leq m, m \in N$, can be given as [10]:

$$
£\left[\left(\frac{d^{\alpha} f}{d t^{\alpha}}\right)(t)\right]=\frac{s^{m} F(s)-s^{m-1} f(0)-s^{m-2} f^{\prime}(0)-\ldots-f^{m-1}(0)}{s^{m-\alpha}} .
$$

\section{Methodology}

In the present procedure by the application of Lemma 1.2 to equation (1.1), when $\alpha=3 / 2$, we get

$$
\widehat{V}(s)=\left[a_{1} s^{2}+b_{1} s^{\frac{3}{2}}+c_{1}\right]^{-1} G(s),
$$

where

$$
G(s)=\left[\left(a_{1} s^{\frac{3}{2}}+b_{1} s\right) v(0)+\left(a_{1} s^{\frac{1}{2}}+b_{1}\right) v^{\prime}(0)\right] s^{-\frac{1}{2}}+\widehat{H}(s)
$$

and

$$
\widehat{H}(s)=£(h(t)) .
$$

Now using inverse Laplace transform formula, we get

$$
v(t)=£^{-1}(\widehat{V}(s))=\frac{1}{2 \pi \iota} \int_{\Gamma} e^{s t} \widehat{V}(s) d s, R(s)>0 .
$$

where $\Gamma$ represents the path of integration, which satisfy $s=s(u)$ given by equation (3.1). Using equation (3.1) in (2.4), the solution $v(t)$ may be given as an integral in $u$,

$$
v(t)=\frac{1}{2 \pi \iota} \int_{\Gamma} e^{s(u) t} \widehat{V}(s(u)) s^{\prime}(u) d u
$$

For a quadrature step $k>0$, if we set $s_{j}=s\left(u_{j}\right), s_{j}^{\prime}=s^{\prime}\left(u_{j}\right)$, where $u_{j}=j k$ for $-N \leq j \leq N$, we get the quadrature formula

$$
v_{N}(t)=\frac{k}{2 \pi \iota} \sum_{j=-N}^{N} e^{s_{j} t} \hat{V}\left(s_{j}\right) s_{j}^{\prime}
$$

To find approximate solution $v_{N}$, we solve system of $2 N+1$ equations $(2.7)$ where, $-N \leq j \leq N$.

$$
\hat{V}\left(s_{j}\right)\left[a_{1} s_{j}^{2}+b_{1} s_{j}^{\frac{3}{2}}+c_{1}\right]=G\left(s_{j}\right)
$$

\section{Contour of integration}

We remarked that the numerical solution $\hat{V}\left(s_{j}\right)$ determined the approximate solution (2.6) for all $t>0$. In practice, accuracy of the approximate solution depends on the use of optimal contour $\Gamma$. A number of such contour available one such path is due to [5] given as

$$
s=\omega+\lambda(1-\sin (\delta-i u)), \quad\left(C_{1}\right)
$$


Very recently the authors [4] proposed two new types of hyperbolic and parabolic contour, which circumvented the slow decay due to Talbot [7]. The parabola is parameterized as

$$
s=\mu(i u+1)^{2},-\infty<u<\infty, \quad\left(C_{2}\right)
$$

while the hyperbola is redefined as

$$
s=\mu(1+\sin (i u-\sigma)),-\infty<u<\infty, \quad\left(C_{3}\right) .
$$

For both the path of integration the parameter $\mu$ uses to controls width of the path, while the parameter $\sigma$, is using to adjust the asymptotic angle. It is shown in the work of [4] the parabolic path (3.2), exhibit the geometric convergence rate, namely

$$
E_{N} \approx O\left(8.12^{-N}\right), \text { when } N \rightarrow \infty
$$

while in the case of hyperbolic contour (3.3), the convergence rate is

$$
E_{N}=O\left(10.2^{-N}\right), \text { when } N \rightarrow \infty
$$

\section{Numerical examples}

In case of practically relevant and more applied problem, we always forced to use some numerical method, when analytical method is not available. In this section we validate our numerical scheme for the following relevant and important problems.

Example 1. Here we apply the present numerical method to the Bagley-Torvik equation [11],

$$
a_{1} \frac{d^{2} v}{d t^{2}}+b_{1} \frac{d^{\alpha} v}{d t^{\alpha}}+c_{1} v(t)=h(t), 0<t \leq 1
$$

In this case we choose the parameters values as $a_{1}=1, b_{1}=1, c_{1}=1$. The function $h(t)=1+t$, and the fractional order $\alpha=\frac{3}{2}$, and the initial conditions as $v(0)=1$ and $v^{\prime}(0)=1$. The exact solution for this problem is $v(t)=1+t$. We apply Laplace transform based-numerical scheme to obtain the numerical solution. We used the three different types of contours $C_{1}, C_{2}$ and $C_{3}$ for approximating the problem (4.1). In this computations we used different types of quadrature points $2 N+1$. We compared our results with an another method [11], we can see that the present numerical scheme for BT equation achieved better accuracy. These results have been shown in Figure(1) and in Table (1). From above the discussion we observed that for a specific range of $N$ the different paths have their own importance. To avoid the computations cost, we use path $C_{3}$, while for better accuracy and using low quadrature order we must use path $C_{2}$, while this have been keenly observed that for long computations we can find more than better accuracy via path $C_{1}$. 


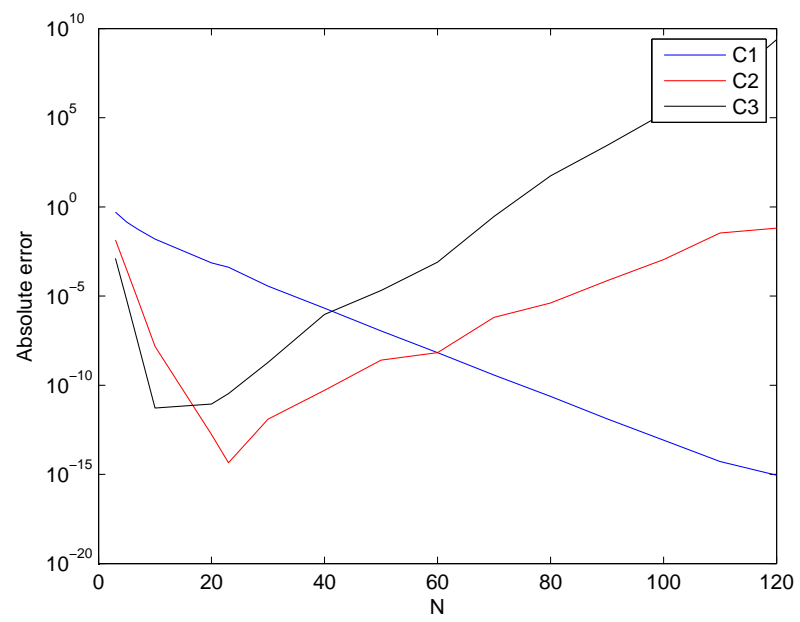

Figure 1. Numerical solution: Plots of absolute error versus N, for the given three types of contours corresponding to problem (4.1).

Example 2. we consider the Bagley-Torvik equation [12],

$$
a_{1} \frac{d^{2} v}{d t^{2}}+b_{1} \frac{d^{\alpha} v}{d t^{\alpha}}+c_{1} v(t)=h(t), 0 \leq t \leq 1
$$

For this problem we choose different values of parameters, $a_{1}=1, b_{1}=c_{1}=0.5$, function $h(t)=8$, and $\alpha=\frac{3}{2}$, and the initial conditions as $v(0)=v^{\prime}(0)=0$. The present LT based numerical scheme is applied to obtain the numerical solution of the problem. The same optimal paths and optimal parameters corresponding to the three paths $C_{1}, C_{2}$ and $C_{3}$ haven been used. The results are shown in Table 2, it can observed that the present LT numerical scheme produced better results than the results obtained in [12]. 


\begin{tabular}{cccc}
\hline $\mathrm{N}$ & Abs. error $\left(\mathrm{C}_{1}\right)$ & Abs. error $\left(\mathrm{C}_{2}\right)$ & Abs. error $\left(\mathrm{C}_{3}\right)$ \\
\hline 5 & $1.40 \mathrm{e}-001$ & $2.97 \mathrm{e}-004$ & $5.7908 \mathrm{e}-006$ \\
7 & $5.50 \mathrm{e}-002$ & $5.95 \mathrm{e}-006$ & $2.3164 \mathrm{e}-008$ \\
10 & $1.57 \mathrm{e}-002$ & $1.51 \mathrm{e}-008$ & $5.2944 \mathrm{e}-012$ \\
20 & $7.31 \mathrm{e}-004$ & $1.74 \mathrm{e}-013$ & $8.7907 \mathrm{e}-012$ \\
23 & $4.12 \mathrm{e}-004$ & $4.53 \mathrm{e}-015$ & $3.3646 \mathrm{e}-011$ \\
30 & $3.60 \mathrm{e}-005$ & $1.25 \mathrm{e}-012$ & $1.9011 \mathrm{e}-009$ \\
40 & $2.06 \mathrm{e}-006$ & $5.18 \mathrm{e}-011$ & $9.2017 \mathrm{e}-007$ \\
50 & $1.10 \mathrm{e}-007$ & $2.53 \mathrm{e}-009$ & $2.0542 \mathrm{e}-005$ \\
120 & $8.94 \mathrm{e}-016$ & $6.42 \mathrm{e}-002$ & $2.4019 \mathrm{e}+009$ \\
\hline$[11]$ & $1.2700 \mathrm{e}-005$ & & \\
\hline
\end{tabular}

TABLE 1. LT Numerical solution: $\left(C_{1}\right)$ related parameters, $t=0.1, \theta=0.1, \delta=0.3812, w=$ $02, r=0.3431$ and $t_{0}=0.01, T=1:\left(C_{2}\right)$ related parameters $\mu=\pi N / 12 t, k=3 / N$. $\left(C_{3}\right)$ related parameters $\sigma=1.1721, A_{\sigma}=\cosh ^{-1}(2 \sigma /(4 \sigma-\pi) \sin \sigma), k=A_{\sigma} / N, \mu=\left(4 \pi \sigma-\pi^{2}\right) N / A_{\sigma} t$, corresponding to (4.1).

\begin{tabular}{cccc}
\hline $\mathrm{N}$ & Abs. error $\left(\mathrm{C}_{1}\right)$ & Abs. error $\left(\mathrm{C}_{2}\right)$ & Abs. error $\left(\mathrm{C}_{3}\right)$ \\
\hline 10 & $7.7161 \mathrm{e}-003$ & $4.9000 \mathrm{e}-008$ & $1.0000 \mathrm{e}-009$ \\
20 & $1.0552 \mathrm{e}-005$ & $1.0000 \mathrm{e}-009$ & $1.0000 \mathrm{e}-009$ \\
23 & $9.8800 \mathrm{e}-007$ & $1.0000 \mathrm{e}-009$ & $1.0000 \mathrm{e}-009$ \\
50 & $1.0000 \mathrm{e}-009$ & $1.0000 \mathrm{e}-009$ & $1.0000 \mathrm{e}-009$ \\
60 & $1.0000 \mathrm{e}-009$ & $1.0000 \mathrm{e}-009$ & $2.9000 \mathrm{e}-008$ \\
90 & $1.0000 \mathrm{e}-009$ & $2.0000 \mathrm{e}-009$ & $1.1000 \mathrm{e}-001$ \\
100 & $1.0000 \mathrm{e}-009$ & $1.3700 \mathrm{e}-007$ & $9.5387 \mathrm{e}-000$ \\
\hline$[12]$ & $1.932 \mathrm{e}-006$ & & \\
\hline
\end{tabular}

TABle 2. LT Numerical solution: $\left(C_{1}\right)$ related parameters, $t=0.1, \theta=0.1, \delta=0.3812, w=$ $02, r=0.3431$ and $t_{0}=0.01, T=1:\left(C_{2}\right)$ related parameters $\mu=\pi N / 12 t, k=3 / N$. $\left(C_{3}\right)$ related parameters $\sigma=1.1721, A_{\sigma}=\cosh ^{-1}(2 \sigma /(4 \sigma-\pi) \sin \sigma), k=A_{\sigma} / N, \mu=\left(4 \pi \sigma-\pi^{2}\right) N / A_{\sigma} t$, corresponding to $(4.2)$.

\section{Conclusion}

In the present work we constructed Laplace transform based numerical scheme for Bagley-Torvik equation. The LIT have been carried out by quadrature rule equipped with optimal paths of integrations. We conclude that this technique is very useful tool for approximating the solution of the Bagley-Torvik equation with high accuracy. From comparison we have observed that the corresponding methodology is more efficient for approximating the solution of Bagley-Torvik equation than other various methods. Therefore we finally conclude that by better selection of quadrature and contour we can improve the corresponding LT technique. 


\section{Bibliography}

[1] K. Diethelm and N. J. Ford, Analysis of fractional differential equations, Journal of Mathematical Analysis and Applications 265 (2002), 229-248.

[2] K. B. Oldham and J. Spanier, The fractional calculas, Mathematics in Science and Engineering, Vol, 111, Academic Press, New York/London, 1974.

[3] D. Sheen, I. H. Sloan and V. Thomee, A parallel method for time discretization of parabolic equations based on Laplace transformations and quadrature, IMA J. Numer. Anal. 23 (2003), 269-299.

[4] J. A. C. Weideman and L. N. Trefethen, Parabolic and hyperbolic contours for computing the Bromwich integral, Mathematics of Computation 76 (2007), 1341-1356.

[5] W. Mclean, V. Thomee, Numerical solution via laplace transforms of a fractional order evolution equation, Journal of Integral Equations and Applications 22 (2010), 57-94.

[6] M. Rizzardi, A modification of Talbot's method for the simultaneous approximation of several values of the inverse Laplace transform, ACM Trans. Math. Software 21 (1995), 347-371.

[7] A. Talbot, The accurate numerical inversion of Laplace transform, J. Inst. Math. Appl. 23 (1979), 97-120.

[8] P. J. Torvik and R. L. Bagley, On the appearance of the fractional derivative in the behavior of real materials, Journal of Applied Mechanics 51 (2) (1984), 294-298.

[9] I. Podlubny, Fractional differential equations: an introduction to fractional derivatives, fractional differential equations, to methods of their solution and some of their applications, Academic Press 198 (1998).

[10] S. Kazem, Exact solution of some linear fracional differential equations by laplace transform, International Journal of Nonlinear Science, 16 (1) (2013), 3-11.

[11] M. A. Z. Raja, J. A. Khan, and I. M. Qureshi, Solution of fractional order system of Bagley Torvik equation using evolutionary computational intelligence, Hindawi publishing corporation, Mathematical Problems in Engineering, (2011), 1-9.

[12] Y. Cenesiz, Y. Keskin and A. Kurnaz, The solution of the Bagley-Torvik equation with the generalized Taylor collocation method, Journal of the Franklin Institute 347 (2) (2010), 452466.

[13] H. M. Srivastava, and Z. Tomovski, Fractional calculus with an integral operator containing a generalized Mittag-Leffler function in the kernel, Applied Mathematics and Computation 211 (1) (2009), 198-210.

[14] A. A. Kilbas, H. M. Srivastava, and J. J. Trujillo, Theory and Applications of Fractional Differential Equations, North-Holland (Elsevier) Series (2006), xvi +523 